\title{
The breakup of a laminar liquid jet by coaxial non-swirling and swirling air streams
}

\author{
Yifan Liang*1, Lars Christian Johansen ${ }^{1}$, Mark Linne ${ }^{1}$ \\ ${ }^{1}$ Institute for Multiscale Thermofluids, School of Engineering, University of Edinburgh, \\ Edinburgh, United Kingdom \\ *Corresponding author email : s1678791@ed.ac.uk
}

\begin{abstract}
This paper describes a preliminary study on shear-based spray formation. A laminar liquid jet is ejected inside co-annular non-swirling and swirling air streams respectively. The aerodynamic Weber numbers of the flow cases range from 4 to 121. In terms of the co-annular swirling jet, the swirl number of the flow cases is equal to 1.2 because the critical swirl number of the nozzle is 0.8. High-speed shadowgraphy is utilised to obtain data on the first droplet locations, breakup lengths of the liquid jet, and two-dimensional wave spatiotemporal spectra for the jets. In order to detect the large-scale instabilities of the central liquid jet, proper orthogonal decomposition (POD) is performed on the high-speed shadowgraphic images.
\end{abstract}

\section{Keywords}

shear jets, jet instability, breakup lengths, droplets

\section{Introduction}

Spray systems are widely used in many areas of life such as the injector of internal combustion (IC) engine, for coating surfaces, and drug delivery to human air passages, for example. There are many types of atomizers such as pressure, rotary, air-assist, air blast atomizers, etc. The liquid jet will experience a pressure drop and then break into drops and ligaments after it leaves the nozzle [1]. That process is called atomization. The size and velocity distribution of the droplets plays a key role in the spray system performance. It is therefore necessary to gain better understanding of how the droplets and ligaments are formed in the spray. Hence, to make the best use of a spray system, it is important to make the process of formation of droplets and ligaments controllable.

Unfortunately, there is currently no fully predictive model for realistic spray formation owing to the lack of detailed spray formation statistics. In general, there are three major breakup mechanisms including turbulence in the liquid, the aerodynamic forces acting on the gas-liquid interface (which are also called "shear forces") and cavitation inside the nozzle [2]. Hence, in order to develop a database, each of these breakup mechanisms should be isolated from the others. Otherwise, atomization might be simultaneously caused by interaction of these three breakup mechanisms. For example, in this paper, in order to separate the shear forces from the other breakup mechanisms, the liquid flow is laminar, and the liquid delivery tube is long and straight to ensure that there is no cavitation inside the nozzle.

The breakup length is one of the most important characteristics of the co-annular nonswirling and swirling jets. In terms of the co-annular non-swirling jet, the breakup length of the liquid jet is related with the momentum flux ratio ( $\mathrm{M}$, (gas momentum)/(liquid momentum) here). When $\mathrm{M} \ll 1$, the breakup length is determined by the liquid jet [3]. On the other hand, when $M \gg 1$, the breakup length depends more on the gas jet [4, 5]. If $\mathrm{M}$ exceeds the critical momentum flux ratio $M_{c}$ (of about 50 ), a gas cavity is formed by the recirculating gas motion downstream of the liquid core. This gas cavity breaks the liquid core, and the breakup length becomes very short [6]. Research on the breakup length of the co-annular non-swirling jets has already been performed by several groups. Lasheras et al. [5] ejected a water jet inside a 
co-annular air jet, and established an entrainment model to describe qualitatively the liquid core length. The aerodynamic Weber numbers (nondimensional numbers are defined in the Nomenclature section) of their experiments ranged from 16 to 800 . To analyse theoretically the breakup length of a co-annular non-swirling jet, Lasheras and Hopfinger [6] started with the dynamic pressure balance at the liquid-gas interface, producing a correlation for the liquid jet breakup length. Farago and Chigier investigated the breakup of a round liquid jet with a non-swirling coaxial air stream [7]. Their aerodynamic Weber numbers and liquid Reynolds numbers ranged from 0.001 to 600 and from 200 to 20000 , respectively. They obtained a correlation for breakup length as well.

Although research on the breakup length of the co-annular non-swirling jets has been done, only a handful of studies on the breakup length of the co-annular swirling jets have been reported. Kumar and Sahu [8] obtained an empirical correlation for the turbulent liquid breakup length in a co-annular swirling jet. Dunand et al. [9] used a Phase Doppler Analyzer (PDA), tomography, and an optical fiber probe to investigate the breakup of a central water jet by a co-annular swirling air jet. They found that the hollow-cone spray appears when the gas swirl number goes beyond the critical swirl number of their nozzle. Furthermore, a transition to "explosive breakup" of the liquid jet (caused by a gas-phase recirculation zone) significantly reduces the breakup length [9]. They also found that the addition of the annular swirling gas stream reduces the breakup length of the liquid jet more significantly as the momentum ratio grows.

Apart from breakup length, shear instability and droplet SMD are the characteristics that are of main interest. Shear instability can be observed on the gas-liquid interface because the aerodynamic forces from the gas act on the liquid jet. Matas et al. [10] investigated shear instabilities in coaxial non-swirling jets using a high-speed camera. They found that, based on the destabilization mechanisms, the shear instability can be categorized into three types; a convective instability, an absolute instability governed by surface tension, and an absolute instability governed by confinement [10]. They also demonstrated that the frequencies of all three instabilities vary as a function of the coflowing gas velocity, and they plotted the frequencies versus gas velocity. Although Matas et al. [10] obtained the frequency spectra of the shear instabilities, the spatial shear instability spectra remain unknown. In this paper, twodimensional spatio-temporal spectra for the co-annular non-swirling and swirling jets are reported.

Hopfinger and Lasheras [4] and Hardalupas and Whitelaw [11] found that the addition of a swirling annular gas stream has a significant effect on the liquid jet breakup if the swirl number goes beyond the critical swirl number. When the swirl number (S) is above the critical swirl number $\left(S_{c r}\right)$, the central recirculating flow and a stagnation point on the centreline of the liquid jet axis can be observed. Hopfinger and Lasheras [4] found a relationship between the critical swirl number and the gas to liquid momentum flux ratio. However, the motion of the annular swirling gas remains unknown. In order to understand how the strength of the air swirl makes a difference to the large-scale instabilities of the co-annular swirling and non-swirling jets, Kumar and Sahu [12] used high-speed shadowgraphy and POD to investigate a central turbulent water jet surrounded by a co-annular air flow with and without swirl, over a wide range of the aerodynamic Weber numbers, $W e_{a}(80-958)$, momenturm ratios, $\mathrm{M}(1-26)$, and swirl numbers, $\mathrm{S}(0-1.6)$. Based on the extracted POD modes, they found three types of largescale instabilities, including jet flapping, wavy or sinuous breakup and explosive breakup, for a wide range of liquid and gas conditions. Kumar and Sahu [12] developed regime maps characterized by swirl number and momentum ratio for a co-annular swirling jet. They found that the strength of the air swirl makes a small difference in the development of the wavy 
breakup instability. However, air swirl enhances the explosive breakup instability in a way that the increase of the strength of the air swirl aids air recirculation.

This paper discusses preliminary experiments on a co-annular (air and water) jet, and a scheme for development of a database on shear breakup. The structure of this paper is organised as follows. Materials and Method talks about the experimental setup, the operating conditions of the atomizer and the experimental procedure. The Results and Discussion section presents preliminary data on the mean values and the standard deviations of the first droplet locations and breakup lengths under various operating conditions. Furthermore, it also presents a two-dimensional spectrum for the co-annular swirling jet. In order to detect the large-scale instabilities of the central laminar liquid jets surrounded by the coaxial swirling/nonswirling air jets, proper orthogonal decomposition (POD) has been performed on the highspeed shadowgrams in this paper.

\section{Materials and Method Overall setup}

The central liquid jet and annular gas stream are water and air respectively. The inner diameters of the liquid and gas tubes are $4 \mathrm{~mm}$ and $10 \mathrm{~mm}$, respectively. The outer diameters of the liquid and gas tubes are $5 \mathrm{~mm}$ and $14 \mathrm{~mm}$, respectively. The air enters the air chamber through an air inlet located at the bottom of the chamber. Then it flows through an air filter to render the nozzle in-flow azimuthally uniform, and then it enters the gas tube (see Figure 1a). In order to introduce swirl into the air, an air swirler is added to the inner surface of the gas tube (see Figure 1b). There are three types of air swirlers with different vane angles. All the air swirlers have four-star shapes. The lengths of the water tubes and gas tubes are $140 \mathrm{~mm}$ and $43 \mathrm{~mm}$, respectively. In order to make sure that laminar flow is fully developed in the liquid jet, the Reynolds number of the central water jet is kept at 480 for all the flow cases.

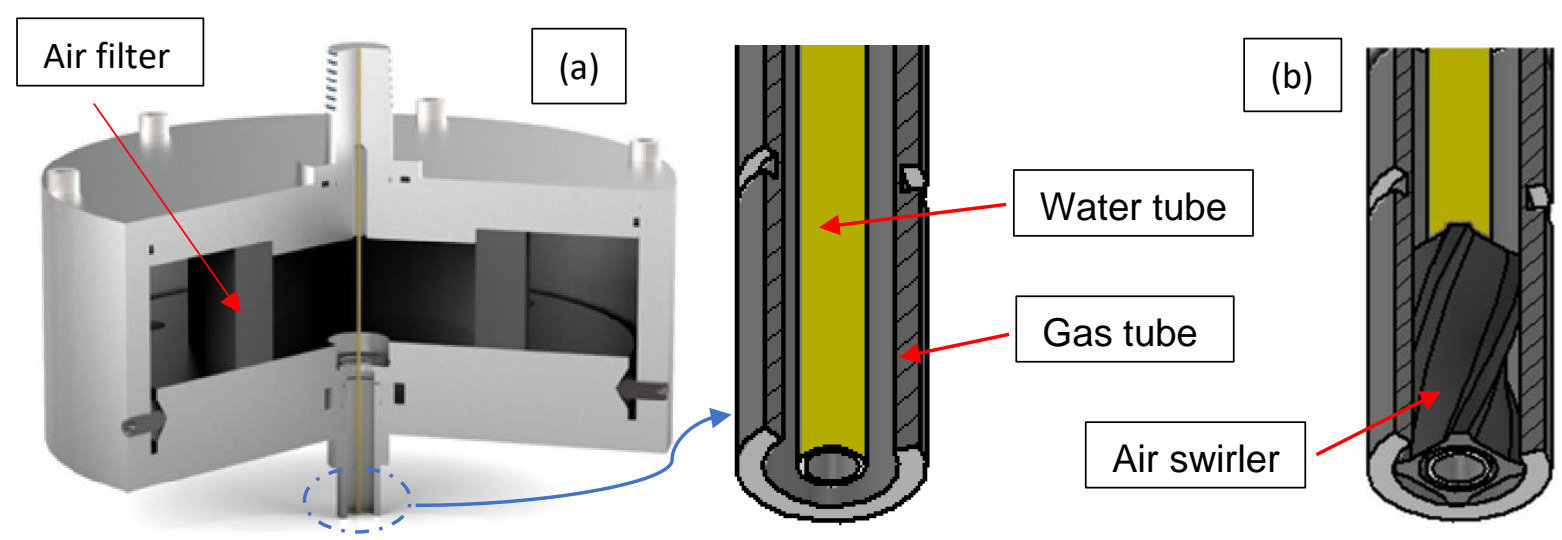

Figure 1 (a) The atomizer and the zoom-in section. The zoom-in section shows the broken-out view of the gas tube without air swirlers attached on it. (b) The broken-out view of the gas tube with air swirler.

\section{Operating conditions for the atomizer}

Five parameters (usually non-dimensional numbers) are generally used to describe the operating conditions of any spray. They include the liquid Weber number, the aerodynamic Weber number, the liquid Reynolds number, the gas Reynolds number, and the momentum flux ratio (these terms are defined in the Nomenclature section).

The swirl number is generally used to describe the operating conditions of a co-annular swirling jet. Details about the correlations for geometrical swirl numbers can be found in Giannadakis et al. [13]. Based on the type of the air swirler used in this paper, the geometrical swirl number is given by, 


$$
S=\frac{2}{3} \tan \theta \frac{1-\left(\frac{D_{0}}{D_{G}}\right)^{3}}{1-\left(\frac{D_{0}}{D_{G}}\right)^{2}}[11],
$$

Where $\theta$ is the swirl vane angle, $D_{0}$ is the external diameter of the liquid tube, and $D_{G}$ is the diameter of the vane pack hub. The vane angles of the air swirlers are $21.1^{\circ}, 45.82^{\circ}$ and $57.06^{\circ}$, which resulted in the geometrical swirl numbers equal to $0.3,0.8$ and 1.2. The operating conditions of the atomizer are shown in Table 1. It should be noted that the operating conditions of the atomizer shown in Table $\mathbf{1}$ are for preliminary study, and $U_{l}$ and $U_{g}$ are the axial bulk velocities of the liquid jet and gas stream at the nozzle exit, respectively. Research on other swirling cases with swirl numbers equal to 0.3 and 0.8 is also planned. The critical swirl number for our nozzle is equal to 0.8 (following Hopfinger and Lasheras. et al. [4]).

Table 1 Operating conditions for the atomizer

\begin{tabular}{|l|l|l|l|l|l|l|l|}
\hline Case & $U_{I}(\mathrm{~m} / \mathrm{s})$ & $U_{g}(\mathrm{~m} / \mathrm{s})$ & $\dot{m}_{g}(\mathrm{~kg} / \mathrm{h})$ & $R e_{I}$ & $W e_{A}$ & $M$ & $S$ \\
\hline 1 & & 7.83 & 2 & & 4 & 6.11 & 0 \\
2 & & 11.75 & 3 & & 9 & 13.77 & 0 \\
3 & & 15.67 & 4 & & 16 & 24.48 & 0 \\
4 & & 17.63 & 4.5 & 438 & 21 & 30.99 & 0 \\
5 & 0.11 & 23.5 & 6 & & 56 & 55.06 & 0 \\
6 & & 29.3 & 7.5 & & 121 & 85.59 & 0 \\
7 & & 43.08 & 11 & & 6 & 9.56 & 0 \\
8 & & 9.79 & 2.5 & & 16 & 24.48 & 1.2 \\
9 & & 15.67 & 4 & 36 & 55.06 & 1.2 \\
10 & & 23.5 & 6 & 81 & 123.89 & 1.2 \\
11 & & 35.25 & 9 & & & & \\
\hline
\end{tabular}

\section{Experimental procedure}

A Phantom VEO 710L high-speed camera acquired images of the swirling and non-swirling jets at 200 frames per second. The resolution of the images is $512 \times 512$ pixels, and the magnification is $0.2 \mathrm{~mm} /$ pixel. There are 17 flow cases. The high-speed camera acquired 99 images for each flow case in this preliminary campaign. Thresholding was applied to each image under various operating conditions. The first droplet locations and the breakup lengths of the liquid jets under various operation conditions were detected using image processing in Matlab. After that, the averages of the breakup lengths and first droplet locations were calculated from among 99 images for each flow case. Furthermore, based on the high-speed images, the interface positions were captured at many different moments in time, and a twodimensional FFT (Fast Fourier Transform) was performed on the data. Corresponding twodimensional spectra were obtained under various operating conditions. POD was performed on these high-speed images as well, to find the dominant instability modes of the liquid jets. The corresponding temporal frequencies of these modes were also obtained by performing FFT on the POD temporal coefficients.

\section{Results and Discussion}

It should be emphasized that the results presented in this section come from a preliminary study. Figure 2 shows the relationships between the normalized mean breakup lengths $\left(L / D_{l}\right) /$ first droplet locations $\left(X_{d} / D_{l}\right)$ and the aerodynamic Weber numbers. The first droplet location $\left(X_{d}\right)$ refers to the axial distance between the first droplet separation and the nozzle exit, and the uncertainty bars are plotted based on the standard deviations of the data. When the aerodynamic Weber number increases, the averages of breakup lengths and the axial 
distances for the first droplet separation reduce, except for the swirling flow cases with low aerodynamic Weber numbers (case 8 and case 9). This can be explained on the basis of the increase of the shear stress acting on the water-air interface, which leads to a more significant destabilization of the liquid jet. However, in terms of the swirling flow cases with low aerodynamic Weber numbers, the breakup length of the co-annular swirling jet tends to increase as the aerodynamic Weber number grows. The swirling annular air stream seems to stabilize the central liquid jet when the aerodynamic Weber number is low. The addition of the swirling annular gas stream has a significant effect on reducing the breakup length of the liquid jet and axial distance for the first droplet separation. This is because the swirling annular air stream destabilizes the liquid jet more significantly compared with non-swirling annular air stream. Research on swirling/non-swirling flow cases with higher momentum ratios and aerodynamic Weber numbers is planned.
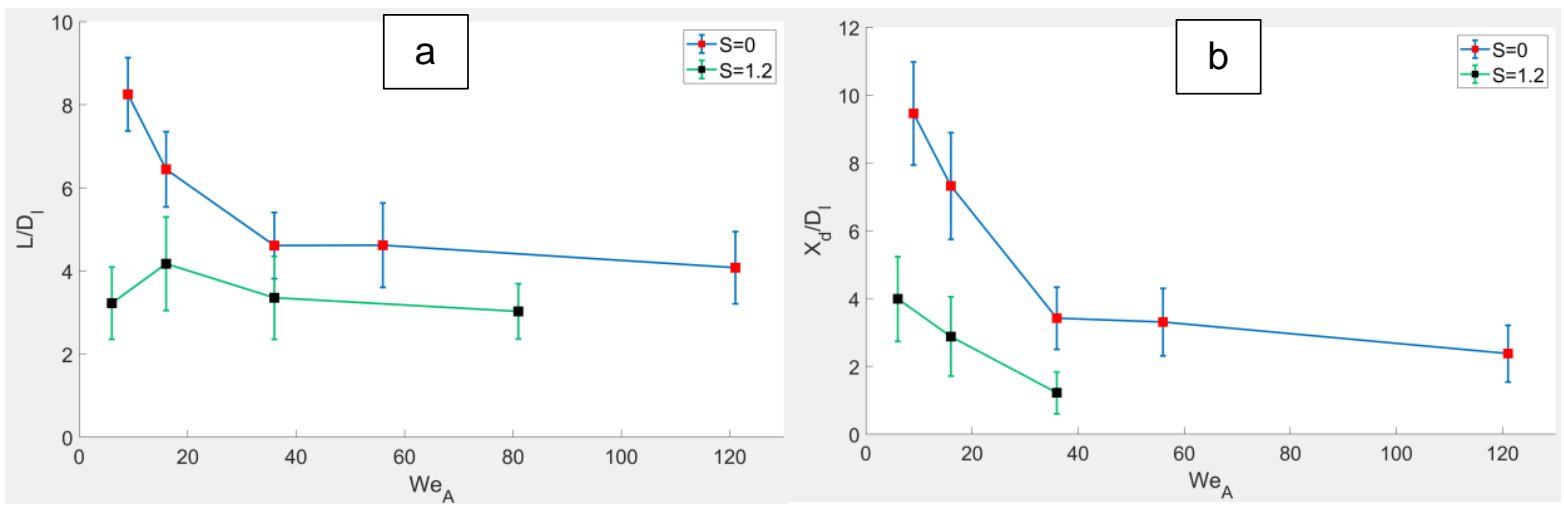

Figure 2 (a) The normalized mean breakup lengths as a function of the aerodynamic Weber numbers. (b) The normalized axial distances for the first droplet separation as a function of the aerodynamic Weber numbers.

The two-dimensional wave spatiotemporal spectrum for case 1 is shown in Figure 3 (this approach is applied only to low air flow cases). The temporal and spatial frequencies corresponding to the peak of the amplitude, are equal to $0.01 \mathrm{~Hz}$ and $0.024 \mathrm{~mm}^{-1}$, respectively. As expected, the primary temporal frequency is very low when the liquid jet is within Rayleigh breakup regime. In order to investigate how the strength of the swirl and aerodynamic Weber number influence the temporal and spatial frequencies, two-dimensional FFT will be performed on other flow cases in the future.
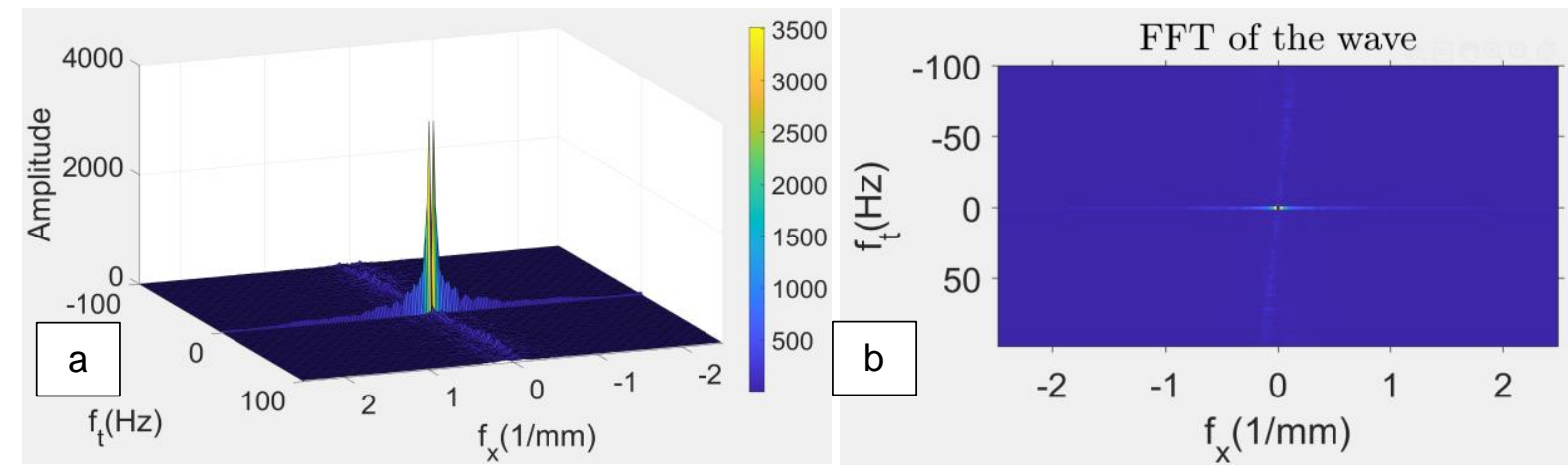

Figure 3 (a) The two-dimensional wave spatiotemporal spectrum for case 1 $\left(\mathrm{We}_{\mathrm{A}}=4, \mathrm{~S}=0\right)$. (b) the top view of the two-dimensional wave spatiotemporal spectrum.

The instantaneous high-speed images, POD modes and the temporal frequencies for case 4 are shown in Figure $\mathbf{4}$ and Figure 5. The flapping instability, which is defined as the lateral 
oscillation of the tail end of the jet, can be observed in Figure 4a. The plot of the singular values shows that the flapping instability can be represented by the first mode, whose singular value is much larger than others (see Figure $\mathbf{4 b}$ ). The temporal frequency of the flapping instability is around $6.675 \mathrm{~Hz}$ (see Figure 5a). In order to investigate how the strength of the swirl and the aerodynamic Weber number influence the temporal frequency of the large-scale instability, the POD analysis on other flow cases is planned.
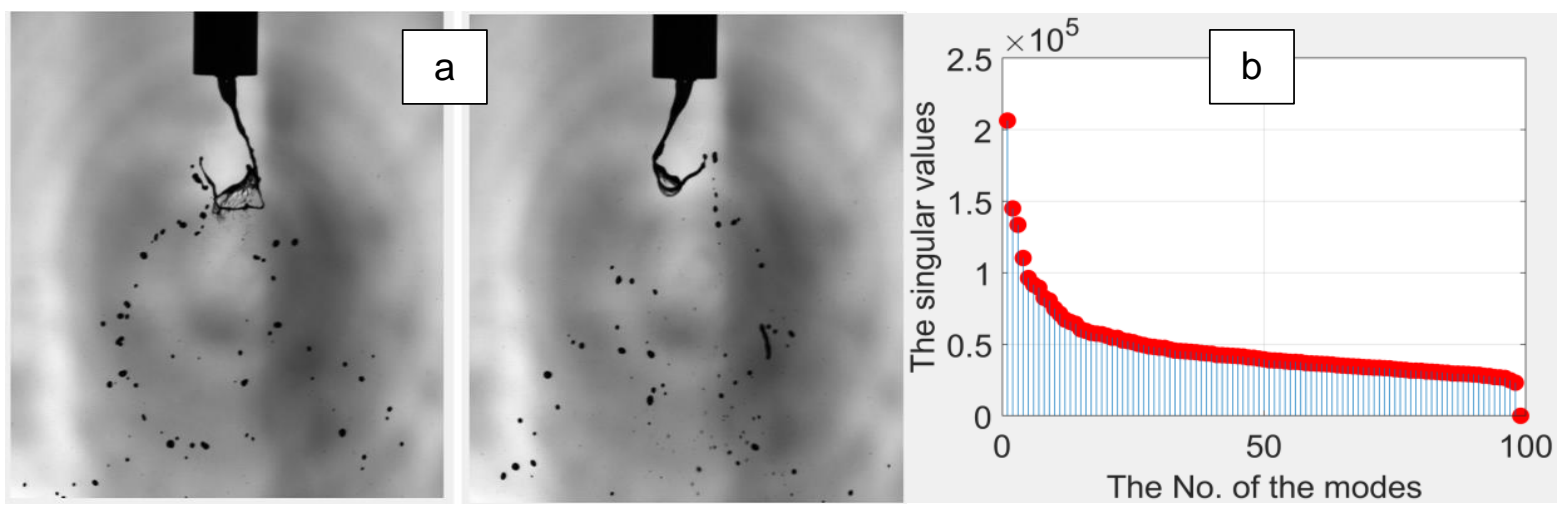

Figure 4 (a) The High-speed shadowgrams for flow case 3. (b) The plot of the singular values for their corresponding modes.
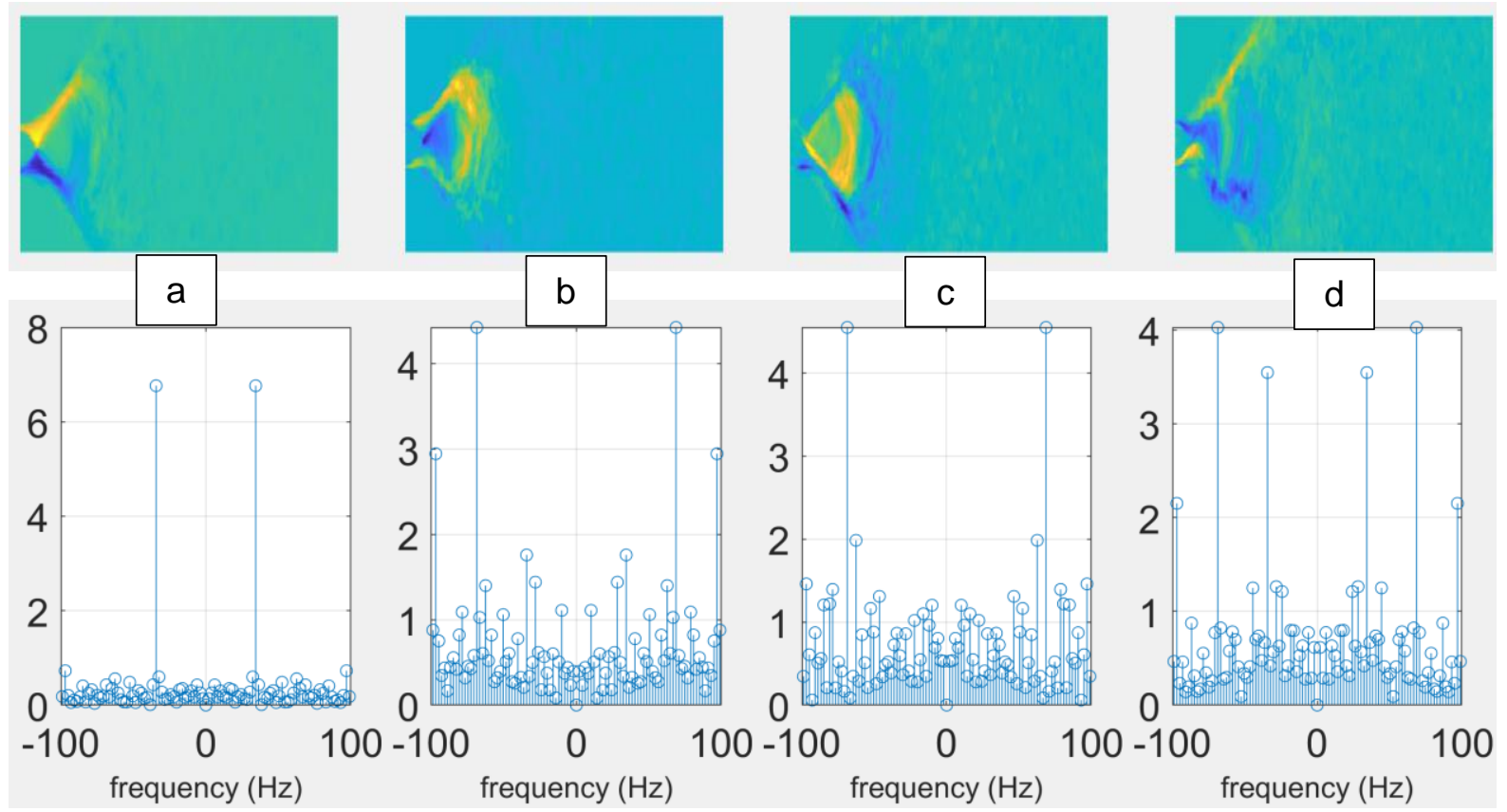

Figure 5 (a) The first principal component (mode) and its temporal spectrum. (b) The second principal component (mode) and its temporal spectrum. (c) The third principal component (mode) and its temporal spectrum. (d) The fourth principal component (mode) and its temporal spectrum.

\section{Conclusions}

When the aerodynamic Weber number increases, the normalized mean breakup lengths and axial distances for the first droplet separation reduce, except for the swirling flow cases with low aerodynamic Weber numbers (case 8 and case 9). In case 8 and case 9 , the normalized mean breakup lengths increase as the aerodynamic dynamic Weber numbers grow. The mechanism behind this phenomenon will be investigated further in the future using PIV for the gas-phase flow. 
When the liquid jet is within the Rayleigh breakup regime, the primary temporal frequency of the instability on the air-water interface is very low. In order to investigate how the strength of the air swirl and momentum ratio influence the primary spatial and temporal frequencies of the instability on the air-water interface, the flow cases with other momentum ratios and swirl numbers will be investigated in the future.

POD analysis for case 4 shows that the dominant large-scale instability mode is a flapping instability. In order to investigate how the strength of the air swirl and momentum ratio influence the dominant instability, POD analysis on other flow cases with high momentum ratios and swirl numbers is planned.

\section{Acknowledgements}

This work was supported in part by EPSRC grants number EP/P020593/1 and EP/P011438/1.

\section{Nomenclature}

$S \quad$ geometrical swirl number $\quad D_{l} \quad$ inner diameter of the liquid tube

$\rho_{l} \quad$ density of the fluid

$\rho_{g} \quad$ density of the gas

$D_{g} \quad$ diameter of the gas tube

$v_{\text {, }}$ kinematic viscosity of the liquid

$A_{g}$ cross sectional area of the gas tube

$U$ relative velocity between the liquid and gas

$W e_{l} \quad$ liquid Weber number $\left(W e_{l}=\rho_{l} U^{2} D_{l} / \sigma_{l}\right)$

$W e_{A}$ aerodynamic Weber number $\left(W e_{A}=\rho_{g} U^{2} D_{l} / \sigma_{l}\right)$

$R e_{l} \quad$ liquid Reynolds number $\left(R e_{l}=U_{l} D_{l} / v_{l}\right)$

$R e_{g} \quad$ gas Reynolds number $\left(R e_{g}=U_{g}\left(D_{g}-D_{l}\right) / v_{g}\right)$

$M \quad$ momentum flux ratio $\left(M=\rho_{g} U_{g}{ }^{2} / \rho_{l} U_{l}^{2}\right)$

$X_{d} \quad$ the axial distance between the first droplet separation and the nozzle exit

\section{References}

[1] J. B. Heywood, Internal Combustion Engine Fundamentals, New York, Chicago, San Francisco, Athens, London, Madrid, Mexico City, Milan, New Delhi, Singapore, Sydney, Toronto: McGraw-Hill Education, 2018.

[2] H. Lefebvre and V. G. McDonell, Atomization and sprays, Boca Raton: CRC Press, 2017.

[3] R. D. Reitz and F. V. Bracco, "Mechanisms of Breakup of Round Liquid Jets," The Encyclopedia of Fluid Mechanics, vol. 3, pp. 223-249, 1986.

[4] E. J. Hopfinger and J. C. Lasheras, "Explosive breakup of a liquid jet by a swirling coaxial gas jet," Physics of Fluids , vol. 8, no. 7, pp. 1696-1698, 1996.

[5] J. C. Lasheras, E. Villermaux and E. J. Hopfinger, "Break-up and atomization of a round water jet by a high-speed annular air jet," Fluid Mechanics, vol. 357, pp. 351379, 1998.

[6] J. C. Lasheras and E. J. Hopfinger, "Liquid jet instability and atomization in a coaxial gas stream," Annual Review of Fluid Mechanics , vol. 32, pp. 275-308, 2000.

[7] Z. Farago and N. Chigier, "Morphological classification of disintegration of round liquid jets in a coaxial air stream," Atomization Sprays, vol. 2, pp. 137-153, 1992.

[8] A. Kumar and S. Sahu, "Optical visualization and measurement of liquid jet core in a coaxial atomizer with annular swirling air," J. Flow Visualization Image Process, vol. 25, pp. 229-244, 2018. 
[9] A. Dunand, J. L. Carreau and F. Roger, "Liquid jet breakup and atomization by annular swirling gas jet," Atomization and Sprays, vol. 15, pp. 223-247, 2005.

[10] J. P. Matas, A. Delon and A. Cartellier, "Shear instability of an axisymmetric air-water coaxial jet,” J. Fluid Mech, vol. 843, pp. 575-600, 2018.

[11] Y. Hardalupas and J. H. Whitelaw, " Coaxial airblast atomizers with swirling air stream," Recent advances in spray combustion: measurements and model simulation, vol. 2, pp. 201-232, 1998.

[12] A. Kumar and S. Sahu, "Large scale instabilities in coaxial air-water jets with annular air swirl," Physics of Fluids, vol. 31, no. 124103, 2019.

[13] A. Giannadakis, K. Perrakis and T. Panidis, "A swirling jet under the influence of a coxial flow," Experimental Thermal and Fluid Science, vol. 32, pp. 1548-1563, 2008. 